\title{
Esquemas de trasmisión de datos en una Microrred a través de una Infraestructura de medición avanzada
}

\section{Data Transmission Schemes in Microgrids through Advance Metering Infrastructure}

\author{
E.E Gaona Garcia ${ }^{1}$, T Morales Vega ${ }^{2}$. C.L Trujillo Rodríguez ${ }^{3}$, F. Santamaría ${ }^{4}$ \\ ${ }^{1}$ GITUD. Universidad Distrital Francisco José de Caldas, Colombia, egaona@udistrital.edu.co \\ ${ }^{2}$ Universidad Distrital Francisco José de Caldas, Colombia, tatiana.morales@transmilenio.gov.co \\ ${ }^{3}$ Universidad Distrital Francisco José de Caldas, Colombia, cltrujillo@udistrital.edu.co \\ ${ }^{4}$ Universidad Distrital Francisco José de Caldas, Colombia, fsantamariap@udistrital.edu.co
}

Recibido: abr 24, 2016. Aceptado: jun 12, 2016. Versión final: jun 23, 2016

\begin{abstract}
RESUMEN
Recientemente los desarrollos tecnológicos se han enfocado hacia el uso eficiente de la energía a partir de la implantación de redes inteligentes. La gestión de los recursos en estas redes requiere el monitoreo de los dispositivos que hacen parte del sistema, en este sentido una infraestructura de medición avanzada al registrar variables como la tensión, corriente, fase, frecuencia y potencia de los equipos de conversión energética y las cargas permitirán al sistema de gestión tomar decisiones que permitan la activación/desactivación de fuentes y la conexión/desconexión de cargas en una microrred en tiempo real. En este artículo se proponen diversas alternativas para la transmisión de los datos en una microrred, a través de una infraestructura de comunicaciones que cumplan con requerimientos mínimos de seguridad, bajos tiempos de retardo, confiabilidad y escalabilidad a partir de la comparación obtenida de los resultados obtenidos en simulación para medios inalámbricos con la implementación de los estándares IEEE 802.15.4 e IEEE 802.11 en Matlab.
\end{abstract}

Palabras Clave: Infraestructura de Medición Avanzada, Microrred Rural, Topología de Red, Protocolos de encaminamiento reactivos.

\begin{abstract}
Recently technological developments have focused on the efficient use of energy from the implementation of smart grids. Management of resources in these networks requires monitoring of the devices that are part of the system. In this sense, an advanced metering infrastructure to record variables such as the voltage, current, phase, frequency and power of energy conversion equipment and loads allows management systems make decisions for activation/deactivation of sources and connect/disconnect of loads in a microgrid in real time. In this paper, several alternatives for data transmission in a microgrid are proposed, by using a communication infrastructure with minimum safety requirements, low delays, reliability and scalability based on the results obtained by simulation in wireless media for IEEE 802.15.4 and IEEE 802.11 standar implemented in Matlab.
\end{abstract}

Keywords: Advance Metering Infrastructure (AMI), Rural Microgrid, Network Topology, Reactive Routing Protocols.

\section{INTRODUCCIÓN}

En la última década, los sistemas de comunicaciones en redes eléctricas se centran en los medidores inteligentes, colectores de información y otros dispositivos de gestión de consumo de energía. Mediante estos, los usuarios y proveedores del servicio (operadores de red) tienen acceso en tiempo real a la información histórica y actual de las cargas conectadas, el consumo, las perdidas, el flujo de energía y la facturación de los usuarios asociados a la red, comprendido en un marco propuesto como

Este artículo puede compartirse bajo la licencia CC BY-ND 4.0 (https://creativecommons.org/licenses/by-nd/4.0/) E.E Gaona Garcia, T Morales Vega. C.L Trujillo Rodríguez, F Santa María. "Esquemas de trasmisión de datos en una Microrred a través de una Infraestructura de medición avanzada", UIS Ingenierías, vol. 15, no. 2, pp. 85-92, jul-dic 2016,doi:10.18273/revuin.v15n2-2016007. 
infraestructura de medición avanzada (AMI, Advanced Metering Infraestructure) [1].

En condiciones normales de operación los dispositivos que hacen parte de una microrred como los recursos energéticos distribuidos (DER, Distributed Energy Resource), cargas y baterías interactúan según el esquema de gestión en la Central de Control Maestro (CCM) [2], estos dispositivos están dotados de un Nodo Sensor (NS) que recolecta y envía información de generación y consumo al CCM de forma determinística. La CCM retorna instrucciones de activación/desactivación de fuentes DER, conexión/desconexión de cargas y carga/descarga de baterías para una operación autónoma de la microrred [3]. Estos constantes cambios, crean la necesidad de tener una AMI en la microrred, como parte fundamental para el intercambio bidireccional de información entre la CCM y los NS, soportados en las tecnologías de la información y las comunicaciones. Para este propósito se requieren dos tipos de infraestructura de información para el flujo de datos en una microrred. El primero es el flujo de información desde los nodos sensores hacia la CCM, y en segundo lugar entre la CCM y un Data Center (DC) que concentra la información de la operación de otras microrredes. El flujo de información desde los nodos se realiza través de enlaces de comunicaciones sobre la línea de potencia (PLC, Power Line Communication) o a través enlaces inalámbricos utilizando tecnologías como ZigBee, HomePlug, 6LoWPAN, Z-wave, LoRa entre otros [4]-[8]. En cambio en el segundo flujo, se pueden utilizar tecnologías de comunicación de mayor alcance como la telefonía móvil celular (3G, 4G, 5G, LTE), enlaces satelitales, de microondas y fibra óptica [9].

La integración de los sistemas de comunicaciones permite la implementación de arquitecturas de control rentables, estables, seguras, confiables y sostenibles [4]. Para esto los esquemas de comunicaciones propuestos requieren de la evaluación de diversos parámetros claves como lo son el tiempo de retardo y la perdida de datos, teniendo estos últimos gran impacto en los esquemas de control en frecuencia que operan en modo isla [10], también lo es el área de cobertura [11], [12]; el tráfico de datos que permiten medir el desempeño de la red en términos de escalabilidad [13], y confiabilidad [14] al momento en que se enlazan nuevos dispositivos a la red, esto se refleja en el producto ancho de banda-distancia acumulado y la tasa de error en la transmisión de datos. Estos aspectos deben definirse para la adecuada operación y gestión de los recursos en una microrred.

Este artículo se encuentra dividido de la siguiente manera: en la sección 2 se presentan las generalidades y tipos de microrredes, en la sección 3 se presentan las características de la infraestructura de medición avanzada. En la sección 4 se presentan los esquemas de transmisión de datos en el entorno de las AMI, en la sección 5 se presentan la metodología empleada en las simulaciones, en la sección 6 los resultados obtenidos para los medios inalámbricos y finalmente, se presentan las conclusiones en la sección 8 .

\section{GENERALIDADES EN MICRORREDES}

La microrred comprende una parte del sistema de distribución eléctrica en media y baja tensión. Incluye una variedad de recursos energéticos distribuidos (DER, Distributed Energy Resources) tales como generadores distribuidos y unidades de almacenamiento de energía, y diferentes tipos de usuarios finales (cargas eléctricas y/o térmicas), así como equipos de comunicación necesarios para la operación y el manejo de energía en tiempo real del sistema [15]. Esta sirve a una gran variedad de clientes, tales como, edificios de viviendas, entidades comerciales, parques industriales, zonas no interconectadas, etc. [16].

Una microrred tiene la capacidad de importar y exportar de forma flexible energía desde y hacia la red, a partir de diferentes tipos de DER, controlar el flujo de potencia activa y potencia reactiva cumpliendo con los requisitos de calidad exigidos por los usuarios que soporta, y manejar el almacenamiento de energía [16]-[18].

Una microrred puede trabajar interconectada a la red eléctrica $o$ en modo aislado. Para cada modo de funcionamiento las necesidades y los esquemas de control son diferentes. Cuando la microrred está conectada a la red eléctrica de distribución, esta sirve como respaldo, y puede alimentar a las cargas locales y entregar energía a la red. Sin embargo, la característica innovadora de una microrred es su capacidad para operar de forma autónoma cuando hay un corte de energía en la red de distribución de energía eléctrica [19].

Este modo de operación se denomina operación aislada y se considera una isla con generadores y cargas locales. De esta manera, los usuarios pueden recibir un servicio continuo, incluso cuando se producen cortes de energía en la red debido a una avería o mantenimiento

\subsection{Microrredes Urbanas}

El desarrollo de las microrredes en el entorno urbano comprende varias aplicaciones, como el uso de paneles fotovoltáicos en los techos de las viviendas [20], hospitales e industrias [21], [22] y microturbinas en edificaciones de oficinas [23].

\subsection{Microrredes Rurales}

En el mundo, la generación de electricidad cubre la demanda en las grandes poblaciones, dejando a las regiones más apartadas del sistema de interconexión eléctrica sin este servicio, ya sea por causas debidas a los costos asociados para su interconexión, la baja demanda por la densidad poblacional, o el difícil acceso al sitio. Sin embargo, las políticas de gobierno en diferentes naciones 
han generado diversas estrategias para suplir las necesidades de electricidad en estas zonas, permitiendo la inclusión de microrredes eléctricas con el fin de generar energía. En la figura 1 se muestra el esquema de una microrred AC, con la ubicación de los dispositivos AMI para el caso inalámbrico.

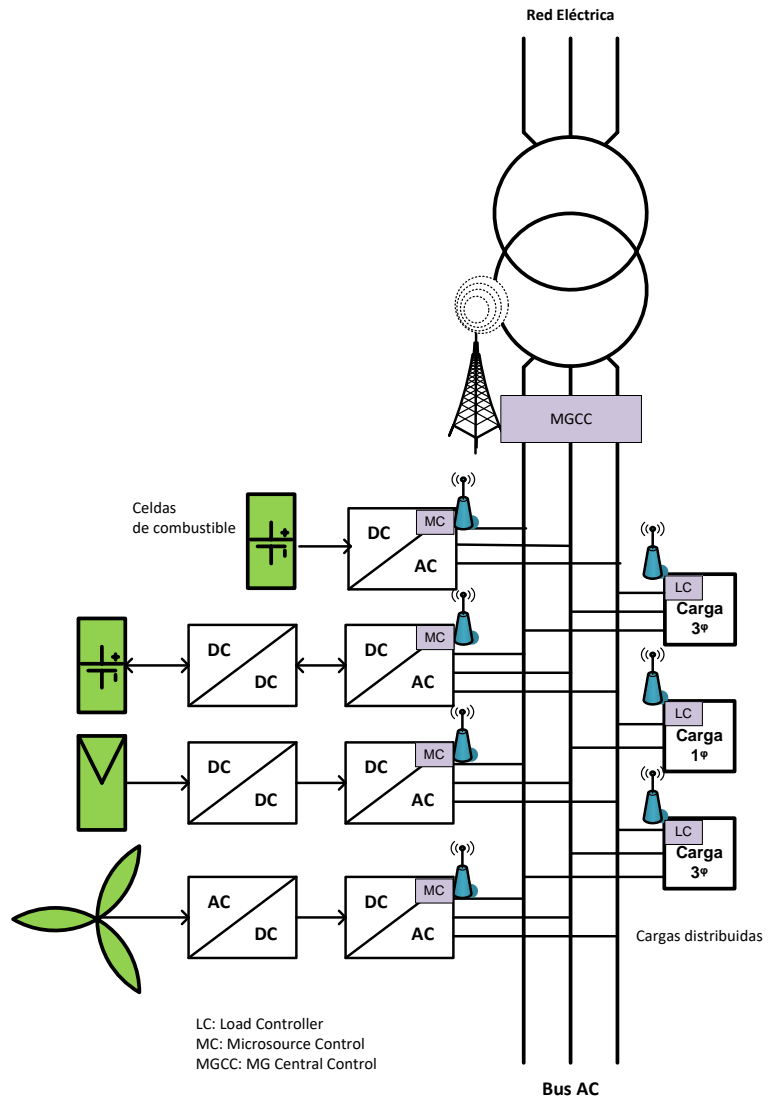

Figura 1. AMI en microrred AC. Fuente: Autores

\section{INFRAESTRUCTURA DE MEDICION AVANZADA}

AMI realiza el registro del consumo y generación de energía en tiempo real, se compone de hardware y software con su sistema de comunicaciones asociado, software de gestión que crea redes bidireccionales entre medidores avanzados y sistemas de utilidad de negocio, permitiendo la recopilación y distribución de información a clientes y otras partes. Estas redes avanzadas de medición pueden usarse para implementar la respuesta a la demanda incluyendo precios dinámicos [24].

\subsection{Nodo Sensor/Actuador}

Los nodos sensores/actuadores recolectan datos de tensión, corriente y fase y frecuencia de las DER, cargas y baterías de la microrred; tienen una capacidad limitada de cómputo y comunicación, debido a esto su tiempo de vida es directamente proporcional a la fuente de alimentación [25], cuentan con un sistema de procesamiento por medio de un microcontrolador que les permite analizar/procesar los datos, almacenarlos temporalmente en memoria y transmitirlos hacia la CCM o hacia otro nodo. También cuentan con un actuador que les permite la activación/desactivación de las fuentes, conexión/desconexión de las cargas al bus AC de la microrred y la carga/descarga de baterías. En el módulo de comunicaciones tienen puertos habilitados que se utilizan para recibir instrucciones/órdenes del nodo Concentrador/Controlador a través de protocolos de transmisión de datos para medios alambrados e inalámbricos. Las partes de un nodo sensor/actuador se pueden observar en la figura 2.

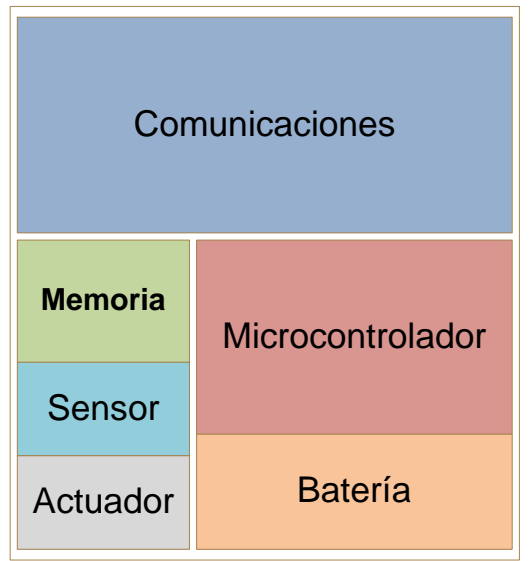

Figura 2. Nodo sensor/actuador de la AMI. Fuente. Elaboración propia

\subsection{Nodo Concentrador/Controlador}

El nodo concentrador/controlador recibe los datos de los nodos sensores/actuadores con el propósito de alimentar el software de gestión de los recursos energéticos de la microrred. Tiene una capacidad de cómputo y almacenamiento de datos más amplia que los nodos sensores/actuadores; aunque su tiempo de vida también es proporcional a la fuente de alimentación, no cuentan con el módulo de sensado y actuador. El resultado de los datos analizados por el software de gestión se envían hacia los nodos sensores/actuadores en forma de instrucciones/ordenes. Este nodo tiene la capacidad de conectarse con la red eléctrica y otras microrredes a través de un medio de transmisión alambrado o inalámbrico. En la figura 3 se pueden observar las partes del nodo concentrador/actuador 


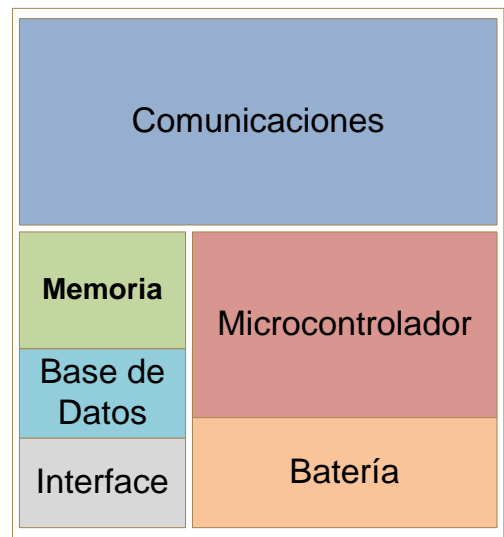

Figura 3. Nodo concentrador/controlador de la AMI. Fuente. Elaboración propia

\subsection{Topologías Soportadas en AMI}

La estructura y topología de la red eléctrica depende de varios factores, como la densidad de población en la zona e incluso la regulación propia del país [26]. Sin embargo, las topologías más comunes se describen a continuación [27].

3.3.1 Topología Urbana. La concentración de los medidores se da en un solo lugar, usualmente en un gabinete de medición, esta topología es típica en áreas de grandes edificaciones. La conexión entre los medidores y la MGCC se realiza a través de líneas físicas con enlaces B-PLC (Broadband PLC).

3.3.2 Topología Rural. A diferencia de la urbana, los medidores están ubicados en su propio gabinete y la distancia entre medidores no es sustancial. En esta topología se ubican viviendas familiares con densidad de población ligera y cercana entre ellas.

3.3.3 Topología Aislada. Representa la estructura donde cada medidor tiene su propio gabinete y la distancia entre medidores adyacentes es sustancial, esta topología es típica para poblaciones de baja densidad como fincas ubicadas en valles y montañas.

\section{ESQUEMAS DE TRANSMISIÓN DE DATOS EN AMI}

Debido al área de cubrimiento y el tamaño limitado de las microrredes eléctricas, los retos que impone el uso de AMI no son tan exigentes como los que se pueden presentar en las aplicaciones industriales, redes de telecomunicaciones y la automatización de procesos. Sin embargo, son fundamentales.

\subsection{Transmisión Inalámbrica}

El diseño de la infraestructura de comunicaciones que emplean enlaces físicos, se torna complejo al aumentar la cantidad de nodos; más aún si las condiciones ambientales donde opera la microrred son adversas, como en los casos de regiones desérticas, selváticas y húmedas; también lo es cuando su despliegue se realiza en zonas de difícil acceso como lo es en zonas aisladas. Por otro lado los medios inalámbricos solucionan problemas de escalabilidad en territorios densamente poblados con espacio limitado. Estas características de alta escalabilidad hacen que las redes de comunicaciones inalámbricas sean un buen candidato para la transferencia de datos en una microrred rural y aislada.

4.1.1 IEEE 802.15.4 del 2006. Especifica la capa física y de acceso al medio para las redes LR-WPAN (Low RateWireless Personal Area Networks). Es un estándar considerado ideal para el monitoreo de múltiples nodos debido a su bajo consumo de energía, bajo costo de despliegue, características de auto-configuración y autoorganización [28]. Dentro de las tecnologías que adoptan este estándar están ZigBee, ZigBEE IP, WirelessHART y 6LowPAN.

4.1.2 IEEE 802.11 de 1999 (Wi-Fi). Opera en la banda ISM de los 2,4 GHz y $5 \mathrm{GHz}$. Entre las ventajas que tiene Wi-Fi están su operación en banda no licenciada y la coexistencia con otras tecnologías que comparten la misma banda de frecuencia, mostrándose robusta en ambientes de RF ruidosos. Sin embargo, presenta un consumo de energía más alto que en el caso de los estándares 802.15.4.

Las características técnicas de los estándares 802.15 .4 y 802.11 se muestran en la Tabla I.

\subsection{Transmisión Alambrada}

Ciertos protocolos se han diseñado para funcionar sobre enlaces físicos como el RS-232, RS-485 e Ethernet (TCP/IP). En el caso de Modbus que es un protocolo orientado al byte, se ha diseñado para la transmisión serial de datos. Muchos controladores y medidores emplean este protocolo por su simplicidad y la funcionalidad Maestro/Esclavo. En cambio DNP3 que también es un protocolo orientado al byte, utiliza capas de aplicación, enlace de datos y una capa de pseudo-transporte. Adicionalmente, este tipo de enlaces tienen requerimientos específicos en términos físicos del cableado, como por ejemplo el uso de dos alambres para el RS-485 y de tres a cinco para el RS-232. 
TABLA I. CARACTERÍSTICAS DE LOS ESTÁNDARES IEEE 802.15.4 Y IEEE 802.11

\begin{tabular}{|c|c|c|}
\hline $\begin{array}{l}\text { Característica/ } \\
\text { Estándar }\end{array}$ & IEEE 802.15.4 & IEEE 802.11 \\
\hline \begin{tabular}{|ll} 
Banda & de \\
Frecuencia & \\
\end{tabular} & $\begin{array}{l}868 \mathrm{MHz} / 915 \mathrm{MHz} \text { y } 2.4 \\
\text { GHz. }\end{array}$ & $\begin{array}{l}2.4 \mathrm{GHz} \text { y } 5 \mathrm{GHz} \\
\text { en la banda ISM }\end{array}$ \\
\hline $\begin{array}{ll}\text { Tasa } & \text { de } \\
\text { transferencia }\end{array}$ & $\begin{array}{l}868 \text { MHz: } 20 \text { kbps; } \\
915 \text { MHz: } 40 \text { kbps; } 2.4 \\
\text { GHz: } 250 \text { kbps }\end{array}$ & $\begin{array}{l}1 \text { Mbps (802.11b) a } \\
1 \text { Gbps (802.11ac) }\end{array}$ \\
\hline Canales & $\begin{array}{l}11 \text { en la banda de } 868 / 915 \\
\text { MHz; } \\
16 \text { en la banda de } 2.4 \mathrm{GHz} \text {. }\end{array}$ & $\begin{array}{l}13 \text { canales } \\
\text { solapados en los } 22 \\
\text { MHz }\end{array}$ \\
\hline Rango & $10-20 \mathrm{~m}$ & $\begin{array}{l}30.5 \mathrm{~m} \text { a } 11 \mathrm{Mbps} \\
92 \mathrm{~m} \text { a } 1 \mathrm{Mbps}\end{array}$ \\
\hline Latencia & Por debajo de $15 \mathrm{~ms}$ & \\
\hline Direccionamiento & Corto 8 bits o 64 bits IEEE & \\
\hline Acceso al canal & $\begin{array}{l}\text { CSMA-CA y CSMA-CA } \\
\text { ranurado }\end{array}$ & CSMA-CA. \\
\hline $\begin{array}{l}\text { Técnica } \\
\text { Modulación }\end{array}$ & DSSS & DSSS \\
\hline
\end{tabular}

Fuente. Elaboración propia

4.2.1 IEEE 1901. Narrowband Power Line Communication (N-PLC). Su uso está orientado a áreas de baja densidad poblacional con una infraestructura cableada bien desarrollada. Los datos se transfieren a través de las líneas de transmisión de potencia empleando portadoras en los rangos de los $100-530 \mathrm{kHz}$, con anchos de banda limitados a $8.5,15,30$ y $86 \mathrm{kHz}$

4.2.2 Broadband Power Line Communication (BPLC). Es una tecnología modulada en el espectro de 2-32 $\mathrm{MHz}$ que es inyectada dentro de las líneas de baja y media tensión, provee comunicaciones con velocidades superiores a los $200 \mathrm{Mbps}$ con enlaces TCP/IP.

4.2.3 IEEE 802.3 (Ethernet). Este estándar puede alcanzar velocidades de 10, 100 y 1 Gbps. Dentro de sus ventajas están su facilidad de instalación y configuración. Como desventajas están el alto costo y los requerimientos de energía que no son convenientes para la conexión de todos los medidores en una red tipo HAN.

\section{METODOLOGÍA}

La evaluación de los esquemas de transmisión de datos inalámbricos en AMI contempla la implementación de los estándares inalámbricos IEEE 802.15.4 e IEEE 802.11 en Matlab. La escogencia de estos estándares se debe a que son ampliamente conocidos, flexibles, escalables y su despliegue e implementación son de bajo costo. El análisis del comportamiento del esquema de transmisión de datos de la red AMI que opera en una microrred requiere de la medición de parámetros básicos de calidad de servicio de la red, como lo son los paquetes transmitidos correctamente en la red de comunicaciones con el flujo neto de información (throughput) y el tiempo empleado para su transferencia con la medición del retardo promedio (delay).

\subsection{Configuración de la Red inalámbrica}

El tamaño del paquete que transmiten los nodos sensores es de 110 bytes, que corresponden a las variables de tensión, corriente, frecuencia y fase medidas en cada dispositivo de la microrred (generadores, cargas, y baterías); la velocidad de transferencia de los datos se determina en 250 kbps según el estándar IEEE 802.15.4 y 6 Mbps según el estándar IEEE 802.11; el tiempo estimado de toma de datos, conversión, procesamiento y envío del paquete ensamblado se configura en $100 \mathrm{~ms}$ para ambos estándares.

\subsection{Topología de red}

Los nodos se ubican en un área de $250 \mathrm{~m}$ x $250 \mathrm{~m}$ con una separación de $100 \mathrm{~m}$ entre nodos en topología estrella, estableciendo así un esquema de gestión centralizada en el nodo concentrador/controlador en la CCM. Estas condiciones son permanentes para ambos estándares.

En la tabla II se resumen los parámetros configurados en la simulación para los estándares IEEE 802.15.4 e IEEE 802.11. 
TABLA II. PARÁMETROS DE SIMULACIÓN PARA EL MEDIO INALÁMBRICO

\begin{tabular}{|c|c|c|}
\hline Tipo de Canal & \multicolumn{2}{|c|}{ Inalámbrico $2.4 \mathrm{GHz}$} \\
\hline Estándar & IEEE 802.15.4 & IEEE 802.11 \\
\hline $\begin{array}{l}\text { Topología de } \\
\text { Red }\end{array}$ & \multicolumn{2}{|c|}{ Estrella } \\
\hline $\begin{array}{l}\text { Modelo } \\
\text { propagación }\end{array}$ & \multicolumn{2}{|c|}{ Dos rayos } \\
\hline Superficie (m) & \multicolumn{2}{|c|}{$250 \times 250$} \\
\hline $\begin{array}{l}\text { Distancia entre } \\
\operatorname{nodos}(\mathrm{m})\end{array}$ & \multicolumn{2}{|c|}{100} \\
\hline $\begin{array}{l}\text { Número de } \\
\text { nodos }\end{array}$ & \multicolumn{2}{|c|}{5 a 19} \\
\hline $\begin{array}{l}\text { Tamaño } \\
\text { paquete (bytes) }\end{array}$ & \multicolumn{2}{|c|}{110} \\
\hline $\begin{array}{l}\text { Velocidad } \\
\text { Transmisión }\end{array}$ & $250 \mathrm{kbps}$ & $6 \mathrm{Mbps}$ \\
\hline $\begin{array}{l}\text { Tasa de envío de } \\
\text { paquetes }\end{array}$ & \multicolumn{2}{|c|}{$100 \mathrm{~ms}$} \\
\hline $\begin{array}{l}\text { Tiempo } \\
\text { simulación }\end{array}$ & \multicolumn{2}{|c|}{$100 \mathrm{~s}$} \\
\hline
\end{tabular}

Fuente. Elaboración propia

\section{RESULTADOS}

Se realizaron simulaciones variando la cantidad de nodos sensores, que representan al grupo de generadores, cargas y baterías. Se iniciaron las simulaciones con 5 nodos, incrementando en pasos de dos nodos hasta completar 19 nodos en topología estrella. La configuración de la red se mantiene igual para ambos estándares y el resto de los nodos.

TABLA III. RESULTADOS ESTÁNDAR 802.15.4

\begin{tabular}{|c|c|c|}
\hline \multirow{2}{*}{$\begin{array}{l}\text { Número } \\
\text { de Nodos }\end{array}$} & \multicolumn{2}{|c|}{ Estándar 802.15.4 } \\
\hline & $\begin{array}{c}\text { Throughput } \\
\text { (kbps) }\end{array}$ & Delay (ms) \\
\hline 5 & 39,0096 & 3,84 \\
\hline 7 & 54,39 & 3,52 \\
\hline 9 & 70,0728 & 3,52 \\
\hline 11 & 84,6972 & 3,52 \\
\hline 13 & 91,2072 & 3,52 \\
\hline 15 & 88,536 & 3,52 \\
\hline 17 & 86,184 & 3,52 \\
\hline 19 & 87,948 & 3,52 \\
\hline
\end{tabular}

\subsection{Estándar IEEE 802.15.4}

En la tabla III se muestran los resultados obtenidos para el estándar IEEE 802.15.4.

En la figura 4 se muestran los resultados obtenidos para el throughput y el retardo promedio (delay) obtenidos con el estándar IEEE 802.15.4. Se simulan los parámetros para diferente cantidad de nodos, se evidencia que el incremento del throughput a medida que aumenta el número de nodos sensores, y se mantiene en la zona de mayor concentración de nodos con un valor promedio de $75.25 \mathrm{kbps}$.

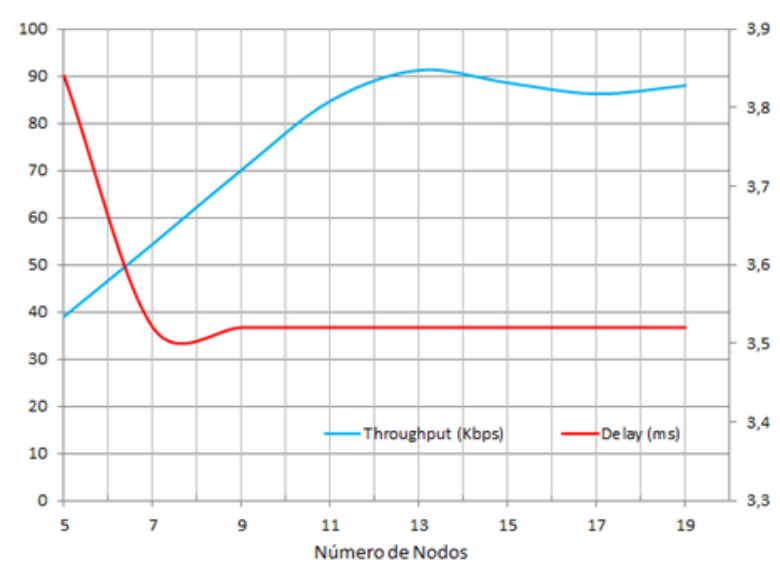

Figura 4. Throughput (kbps) y delay (ms) para el estándar IEEE 802.15.4 en topología estrella. Fuente. Elaboración propia

En cuanto al delay, este parámetro decae drásticamente en la zona de 5 a 7 nodos sensores, y permanece constante a medida que aumenta la cantidad de nodos sensores con un valor constante de $3.52 \mathrm{~ms}$. Esto se debe a que en la red llega a su máxima capacidad en la transferencia de datos, como lo representa un throughput constante al aumentar la cantidad de nodos.

\subsection{Estándar IEEE 802.11}

En la tabla IV se muestran los resultados obtenidos para el estándar IEEE 802.11.

En la figura 5 se muestran los resultados del throughput y el retardo promedio (delay) obtenidos con diferentes cantidad de nodos sensores utilizando el estándar IEEE 802.11. Se aprecia que el throughput sigue aumentando con el número de nodos sensores, siendo mayor al obtenido con el estándar IEEE 802.15.4. Esto se debe a la mayor velocidad de transmisión de datos empleada, sin embargo, el valor promedio del throughput es de 78.68 kbps, siendo levemente superior que el estándar IEEE 802.15.4. Este parámetro se ve afectado debido al bajo throughput en la región de menor cantidad de nodos sensores. 
TABLA IV. RESUlTADOS ESTÁNDAR 802.11

\begin{tabular}{|c|c|c|}
\hline \multirow{2}{*}{$\begin{array}{c}\text { Número } \\
\text { de Nodos }\end{array}$} & \begin{tabular}{c}
$|c|$ \\
Ehroughóndar 802.11 (Wi-Fi) \\
\cline { 2 - 3 } (Kbps)
\end{tabular} & Delay (ms) \\
\hline 5 & 0,15689 & 0,273 \\
\hline 7 & 0,18625 & 0,27 \\
\hline 9 & 7,7314 & 0,269 \\
\hline 11 & 94,6 & 0,2671 \\
\hline 13 & 111,8 & 0,266 \\
\hline 15 & 129 & 0,264 \\
\hline 17 & 135 & 0,261 \\
\hline 19 & 151 & 0,257 \\
\hline \multicolumn{2}{|c|}{ Fuente. Elaboración propia } \\
\hline
\end{tabular}

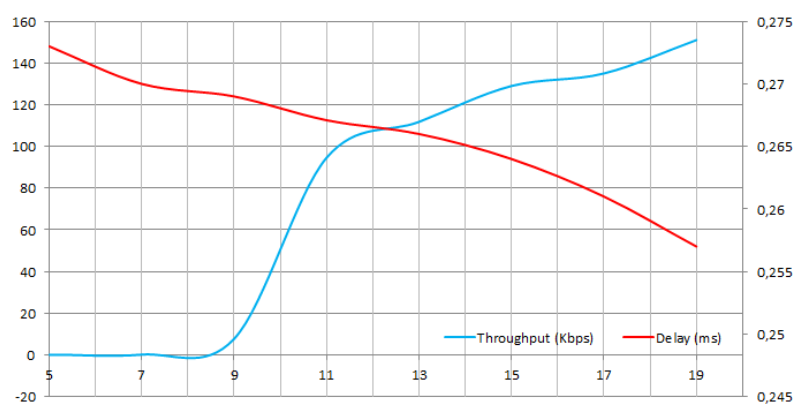

Número de Nodos

Figura 5. Throughput (kbps) y delay (ms) para el estándar IEEE 802.11 en topología estrella. Fuente. Elaboración propia

En cuanto al comportamiento del retardo promedio, se evidencia que es decreciente a medida que aumenta la cantidad de nodos sensores, siendo mucho menor que el presentado en el estándar IEEE 802.15.4, esto también se debe a la alta velocidad en la transferencia de los datos. Las curvas de la figura 5 muestran que no se alcanza la máxima capacidad de transferencia de datos de la red, ya que no se identifica un valor estable para el throughput y el retardo promedio, como si se reflejó con el estándar IEEE 802.15.4, esto muestra que la red admite una mayor cantidad de nodos.

\section{CONCLUSIONES}

Este artículo presenta las diferentes alternativas para el intercambio de datos de control en una microrred, a través de una infraestructura de medición avanzada con requerimientos mínimos de seguridad, bajos tiempos de retardo, confiabilidad y escalabilidad, a partir de las simulaciones obtenidas de los estándares IEEE 802.15.4 e IEEE 802.11 implementados en Matlab.
El diseño de la red de comunicaciones empleando enlaces inalámbricos en microrredes simplifica en gran medida la interconexión de los medidores inteligentes, permite mayor flexibilidad y escalabilidad en el momento en que la red crece; sin embargo, es necesario considerar la calidad del servicio, ancho de banda y latencia al realizar el despliegue cuando aumenta la cantidad de nodos.

En las simulaciones ambos estándares inalámbricos el IEEE 802.15.4 e IEEE 802.11, presentaron retardos promedio bajos, con valores entre 0.257 y $3.52 \mathrm{~ms}$, esto permite la transferencia de valores de referencias e instrucciones a los controladores que operan en la microrred en tiempos cortos sin que se afecte el funcionamiento, logrando así realizar una adecuada gestión de los recursos.

\section{AGRADECIMIENTOS}

Esta investigación es financiada por el CIDC (Centro de Investigación y Desarrollo Científico) Universidad Distrital Francisco José de Caldas in Bogotá D.C.Colombia. Proyecto Numero 2-5-524-16 y cuenta con la participación de los grupos de investigación LIFAE, GCEM y GITUD.

\section{REFERENCIAS}

[1] D. G. Hart, "Using AMI to realize the Smart Grid," in 2008 IEEE Power and Energy Society General Meeting - Conversion and Delivery of Electrical Energy in the 21st Century, 2008, pp. $1-2$.

[2] W. Su and J. Wang, "Energy Management Systems in Microgrid Operations," Electr. J., vol. 25, no. 8, p. 16, 2012.

[3] G. Andria, F. Attivissimo, G. Cavone, A. Di Nisio, and M. Spadavecchia, "Toward a new smart metering paradigm for microgrid," 2013 IEEE Int. Work. Meas. Netw., pp. 79-82, 2013.

[4] A. Bani-Ahmed et al., "Microgrid communications: State of the art and future trends," in 2014 International Conference on Renewable Energy Research and Application (ICRERA), 2014, pp. 780-785.

[5] A. Kwasinski and P. Krein, "A microgrid-based telecom power system using modular multipleinput dc-dc converters," in Telecommunications Conference, 2005. INTELEC'05. TwentySeventh International, 2005, pp. 515-520.

[6] A. Llaria, O. Curea, J. Jimenez, J. L. Martin, and A. Zuloaga, "Wireless communication system for microgrids management in islanding," in Proceedings of the 2011 14th European Conference on Power Electronics and Applications, 2011, pp. 1-10. 
[7] A. Mahmood, N. Javaid, and S. Razzaq, "A review of wireless communications for smart grid," Renew. Sustain. Energy Rev., vol. 41, pp. 248-260, Jan. 2015.

[8] A. G. Phadke and J. S. Thorp, "Communication needs for Wide Area Measurement applications," in 2010 5th International Conference on Critical Infrastructure (CRIS), 2010, pp. 1-7.

[9] C. Pirak, T. Sangsuwan, and S. Buayairaksa, "Recent advances in communication technologies for smart grid application: A review," in 2014 International Electrical Engineering Congress (IEECON), 2014, pp. 1-4.

[10] D. Rua, L. Pereira, Gil N, and J. a. Peças Lopes, "Impact of multi-Microgrid Communication systems in islanded operation," in Innovative Smart Grid Technologies (ISGT Europe), 2011, pp. 1-6.

[11] L. Efraimsson, J. Åhman, and H. Bringsell, "Low loss micro grid for RBS sites," in INTELEC, International Telecommunications Energy Conference (Proceedings), 2011, p. 3.

[12] C. Muller, H. Georg, M. Putzke, and C. Wietfeld, "Performance analysis of radio propagation models for Smart Grid applications," 2011 IEEE Int. Conf. Smart Grid Commun., pp. 96-101, Oct. 2011.

[13] J. Zhou, R. Q. Hu, and Y. Qian, "Scalable distributed communication architectures to support advanced metering infrastructure in smart grid," IEEE Trans. Parallel Distrib. Syst., vol. 23, no. 9, pp. 1632-1642, 2012.

[14] M. Souryal, C. Genti, D. Griffith, D. Cypher, and N. Golmie, "A Methodology to Evaluate Wireless Technologies for the Smart Grid," in 2010 First IEEE International Conference on Smart Grid Communications, 2010, pp. 356-361.

[15] H. Hussein, S. Harb, and N. Kutkut, "Design considerations for distributed micro-storage systems in residential applications," in Telecommunications Energy Conference (INTELEC), 32nd International., 2010, p. 6.

[16] F. Katiraei, R. Iravani, N. Hatziargyriou, and A. Dimeas, "Microgrids management," IEEE Power Energy Mag., vol. 6, no. june, pp. 54-65, 2008.

[17] J. M. Guerrero, S. Member, J. C. Vásquez, J. Matas, M. Castilla, and L. G. De Vicuña, "Control Strategy for Flexible Microgrid Based on Parallel Line-Interactive UPS Systems," vol. 56, no. 3, pp. 726-736, 2009.

[18] C. L. Trujillo Rodriguez et al., "Generalidades de Microrredes Eléctricas," in Microrredes Eléctricas, 1st ed., Bogotá D.C.: Universidad Distrital, 2015, p. 178.

[19] E. E. Gaona García, C. L. Trujillo, and J. A. Guacaneme, "Rural Microgrids and its potential application in Colombia," Renew. Sustain.
Energy Rev., vol. 51, pp. 125-137, 2015.

[20] P. Papageorgas, D. Piromalis, K. Antonakoglou, G. Vokas, D. Tseles, and K. G. Arvanitis, "Smart Solar Panels: In-situ Monitoring of Photovoltaic Panels based on Wired and Wireless Sensor Networks," Energy Procedia, vol. 36, pp. 535545, Jan. 2013.

[21] M. Sechilariu, B. Wang, and F. Locment, "Building-integrated microgrid: Advanced local energy management for forthcoming smart power grid communication," Energy Build., vol. 59, pp. 236-243, Apr. 2013.

[22] A. H. Mohsenian-Rad and A. Leon-Garcia, "Optimal residential load control with price prediction in real-time electricity pricing environments," IEEE Trans. Smart Grid, vol. 1, no. 2, pp. 120-133, 2010.

[23] A. Davigny and B. Robyns, "Variable speed turbine based wind farm including storage system connected to a power grid or islanded," in International Conference on Renewable Energy and Power Quality, ICREPQ, 2006, pp. 5-7.

[24] NIST, Framework and Roadmap for Smart Grid Interoperability Standards, vol. 3. 2014.

[25] S. Escolar, J. Carretero, F. García-Carballeira, and J. Fernandez, "Acabando con los desarrollos Ad-Hoc en Wireless Sensor Networks," in XI Jornadas de paralelismo, 2006.

[26] "Electrical metering - Data exchange meter reading, tariff and load control - Part 21: Direct local data exchange, IEC 62056-21," 2002.

[27] I. Petrusevski, M. Zivanovic, A. Rakic, and I. Popovic, "Novel AMI architecture for real-time Smart Metering," in 2014 22nd Telecommunications Forum Telfor (TELFOR 2014), 2014, pp. 664-667.

[28] A. I. Sabbah, A. El-Mougy, and M. Ibnkahla, “A Survey of Networking Challenges and Routing Protocols in Smart Grids," IEEE Transactions on Industrial Informatics, vol. 10, no. 1. pp. 210221, 2014. 